\title{
Long Term Performance of the Pierre Auger Observatory
}

\author{
Koun Choi ${ }^{* a}$ for the Pierre Auger Collaboration ${ }^{\dagger b}$ \\ ${ }^{a}$ Université Libre de Bruxelles, Brussels, Belgium \\ ${ }^{b}$ Observatorio Pierre Auger, Av. San Martín Norte 304, 5613 Malargüe, Argentina \\ E-mail: auger_spokespersons@fnal.gov \\ Full author list: http://www.auger.org/archive/authors_icrc_2019.html
}

\begin{abstract}
The Pierre Auger Observatory is the largest detector ever built to measure ultra-high energy cosmic rays. It employs a hybrid technique, combining a surface detector consisting of 1660 waterCherenkov stations and a fluorescence detector composed of 27 Schmidt telescopes. The construction of the Observatory started in 2004, and since then, it has been continuously taking data in a stable manner. We will present the behavior of the Observatory over more than 14 years and the expected response into the future with the AugerPrime upgrade now underway. Key performance indicators such as the on-time and the event rates will be presented, along with reference to calibration and monitor instruments.
\end{abstract}

36th International Cosmic Ray Conference - ICRC2019

24 July - 1 August, 2019

Madison, Wisconsin, USA

\footnotetext{
* Speaker.

${ }^{\dagger}$ for collaboration list see PoS(ICRC2019)1177
} 


\section{Overview of the Pierre Auger Observatory}

The Pierre Auger Observatory is the world's largest cosmic ray air-shower detector. The Observatory is built on a $3000 \mathrm{~km}^{2}$ high plain in the province of Mendoza, Argentina. It adopts a hybrid design which combines a Surface Detector (SD) consisting of 1660 water-Cherenkov stations and a Fluorescence Detector (FD) composed of 27 Schmidt telescopes deployed at four different sites overlooking the SD array. The SD captures the lateral spread of air-showers at ground level, while the FD observes their longitudinal profiles.

The construction of the SD started in January 2004, and it has been running in full configuration since 2008. After the completion of the $S D-1500$ array with $1.5 \mathrm{~km}$ spacing between the stations placed on a triangular grid, the low-energy extension of the SD, SD-750 array, was started. It comprises 61 stations with a grid spacing of $750 \mathrm{~m}$ and has been taking data since 2011. An SD station is composed of a water tank of $3.6 \mathrm{~m}$ diameter and $1.2 \mathrm{~m}$ height housing a liner bag made from reflective Tyvek. The liner bag is filled with ultra-pure water, and three 9-inch photomultiplier tubes (PMTs) optically coupled to water look downwards into the tank. When relativistic charged particles from an air shower pass through the inner volume, emitted Cherenkov light is reflected on the inner Tyvek surface and detected by the PMTs. Each station is equipped with a solar power battery and panel, a front-end electronics board, GPS, and a communication antenna for autonomous operation. The Cherenkov light observed by each PMT is digitized at $40 \mathrm{MHz}$ by a 10 bit Flash Analog-to-Digital Convertor (FADC) channels for the output from the last PMT dynode and the anode.

The FD detects nitrogen fluorescence light induced by air-showers in the atmosphere. A single telescope has a field of view of $\left(30^{\circ} \times 30^{\circ}\right)$ in azimuth and elevation angles, with a minimum elevation of $1.5^{\circ}$ above the horizon. The combination of the six telescopes in each site covers $180^{\circ}$ in azimuth angle. A spherical mirror of $3.4 \mathrm{~m}$ radius of curvature focuses light onto a camera composed of 440 hexagonal PMTs assembled in a $(22 \times 20)$ matrix taking data at a frequency of $10 \mathrm{MHz}$. The number of photons detected by each camera pixel is used to evaluate the energy deposited in the atmosphere as a function of depth. Twelve fluorescence telescopes in the Los Leones and Coihueco sites started data taking in January 2004. Another six telescopes entered in acquisition in May 2005 at the Los Morados site, and finally, six telescopes in the Loma Amarilla site became operational in March 2007. Since September 2009, three additional telescopes (HEAT: High Elevation Auger Telescope) with an elevated field of view $\left(30^{\circ} \sim 60^{\circ}\right)$ have also been operating to study lower-energy cosmic rays.

The atmosphere is the medium where the air showers develop and thus an important part of the Observatory is dedicated to measuring the atmospheric conditions and the concentration of aerosols present in the air. The Observatory includes a set of high-quality devices for monitoring clouds and aerosols such as infrared cameras and laser facilities. Details on the design and previous studies on the performance of the Observatory can be found in [1].

Currently, the Observatory is undergoing the AugerPrime upgrade program whose major aim is to enhance the sensitivity of the SD to the mass composition at the highest energies by the deployment of the SSD (Surface Scintillator Detector) on top of the SD stations. The upgrade plan also includes a radio detector deployment, the AMIGA (Auger Muons and Infill for the Ground Array) project, as well as an increase of the duty cycle of the FD [2]. Thus it is important to ensure 


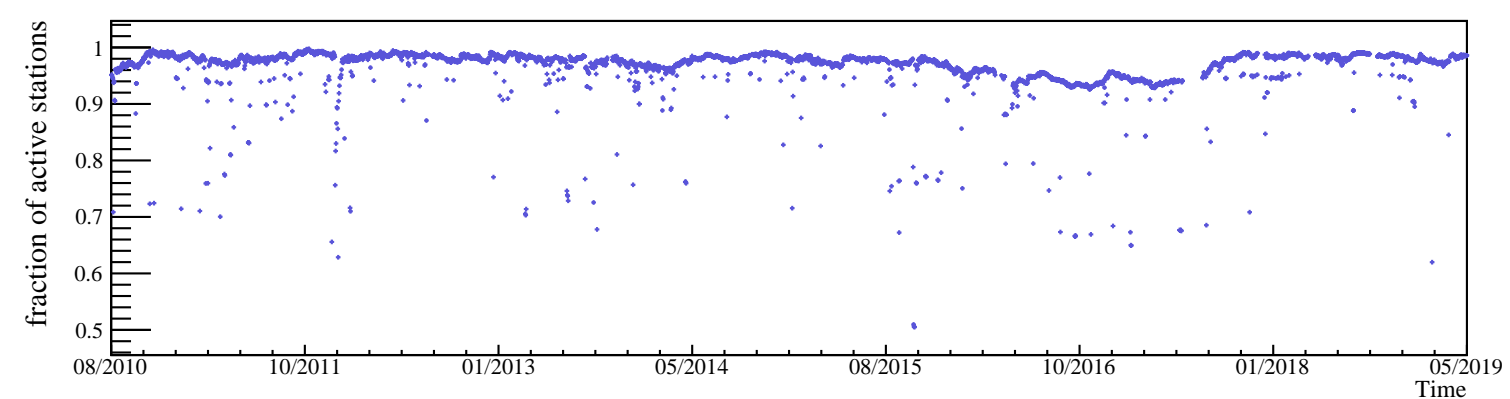

Figure 1: Number of active SD stations normalized to the number of deployed SD stations as a function of time.

that the detectors will continue to take high-quality data over the next decade.

The functioning of the SD, the FD and the other instruments is constantly monitored, from observables related to the PMTs to higher-level variables used in advanced analyses. In this work, we will provide information on the long term performance of the Pierre Auger Observatory by reviewing its behavior over more than 14 years. In section 2, we will describe the performance of the SD and its expected performance in the next decade. We will review the performance of the FD and of the calibration and atmosphere monitoring instruments in section 3, and conclude in section 4.

\section{Long term performance of the Surface Detector}

The SD tank and the electronics are exposed to unstable weather conditions such as a large temperature variation, lightning, high salinity, dust and humidity. These environmental conditions can damage the detector and influence the quality of data. To constantly monitor the detector condition and response, various sensors are installed in every SD station. Variables related to temperature, battery power, PMT voltage and current, and dynode/anode ratio (the ratio of the amplitude of the output from the last PMT dynode to the one from the anode) are sent to the Central Data Acquisition System (CDAS) and then exported to a MySQL database server [1, 3, 4]. Besides monitoring the conditions of the station hardware, the number of triggers a station is transmitting is continuously surveyed. Each station has two levels of triggers (called $T 1$ and T2). Due to the limited data transmission bandwidth, the trigger algorithms are implemented locally in the station software. The T2 trigger selects signals with amplitudes exceeding a threshold (TH), or signals that are spread in time (time over threshold or ToT), and are sent to CDAS to form the trigger for air-showers based on time and spatial coincidence of the signals [5]. The number of active stations able to send T2 signals is constantly monitored. The ratio of active stations to the total number of deployed stations is depicted in Fig. 1. Since the beginning of the deployment, on average more than $95 \%$ of all stations have been functioning. Lower values correspond to DAQ downtime, communication issues in the data transmission to CDAS or other on-site problems occasionally occurring for individual stations. The time fraction when the entire array was not transmitting data was less than $1 \%$ since 2004 .

The particles produced in air showers initiated by low-energy cosmic rays pass through the stations 

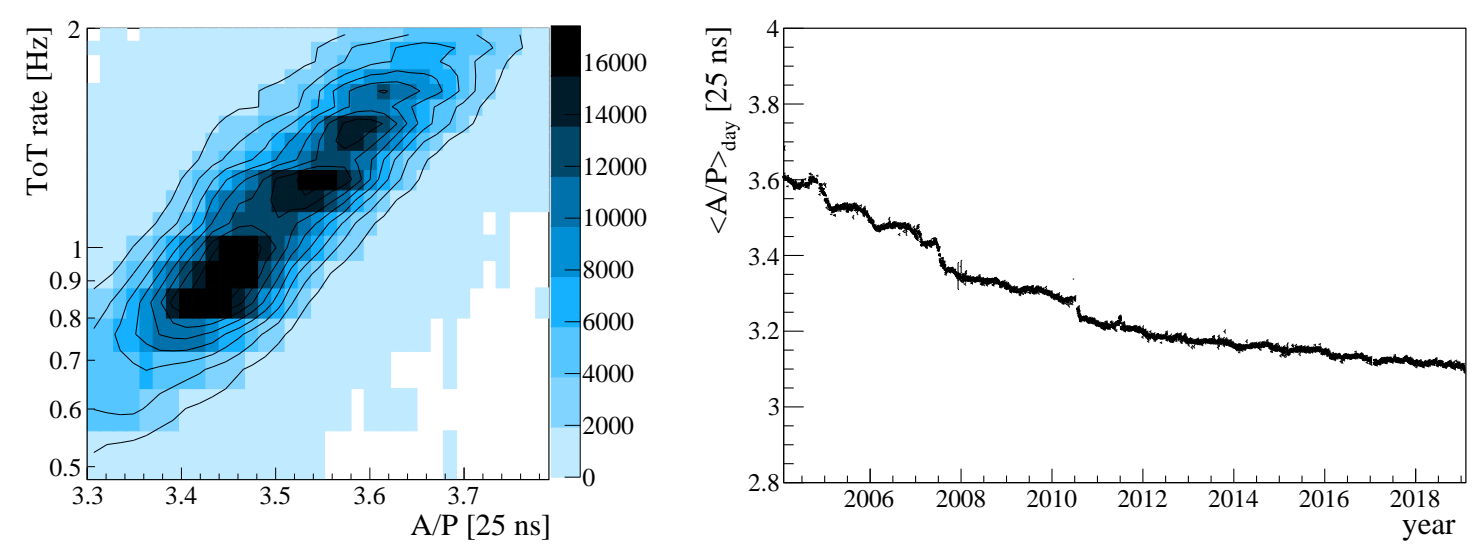

Figure 2: Left: Correlation between A/P and ToT rate plotted using monitoring data from October 2006. Right: Daily average of A/P of one of the three PMTs in the station Rocio (station id 270) as a function of time.

at a rate of $\sim 3000$ per second. Selected by a simple threshold calibration trigger, the amplitude and the charge of each signal are compiled in individual histograms for each PMT. The calibration histograms are filled during one minute. Among the particles entering the tank, the muons produce a larger amount of Cherenkov photons and provide a distinctive larger area and a higher peak than the electrons and photons. These values are computed in the station software and then sent to CDAS every six minutes. Entire calibration histograms are transmitted to CDAS with each triggered airshower. By employing the uniform flux of the atmospheric muons, a uniform calibration for the entire array is achieved.

A good variable to assess and understand the slow changes in the detectors is the area over peak ratio (A/P) of the atmospheric muon signals. This variable is related to the reflectivity of the Tyvek liner and water transparency, as well as the response of PMTs and of the electronics. The correlation with the ToT trigger, which depends on the shape of the muon pulse and therefore on the A/P, is illustrated in Fig. 2 (left). The right plot of Fig. 2 is an example of A/P evolution showing a typical behavior, of one PMT in a station which has been taking data since 2004. After deployment, most stations experience a rapid decrease in $\mathrm{A} / \mathrm{P}$, followed by a milder slope which tends to become flat. An annual modulation related to the seasonal temperature variation is also seen. In 2007 and 2010, some of the stations experienced sudden changes in A/P, which are correlated to very cold winters with temperature drops below $-10^{\circ} \mathrm{C}[6]$. In the last nine years, the freezing temperatures in winters no longer influenced A/P behavior, as can be seen in the winter of 2018, where the temperature dropped below $-10^{\circ} \mathrm{C}$, but no significant change in $\mathrm{A} / \mathrm{P}$ has been observed.

The stacked distribution of the A/P loss is shown in the left plot of Fig. 3. For each A/P profile, the average $\mathrm{A} / \mathrm{P}$ in 2018 is computed, and the $\mathrm{A} / \mathrm{P}$ loss is defined as the ratio of this value to the one at the initial deployment year. Among the 4175 PMTs used in the evaluation, less than $18.5 \%$ of the PMTs have experienced a decrease of more than $15 \%$ compared to their initial values. Shown in the same plot is the stacked distribution of the A/P loss since 2013. In the last five years, A/P has stabilized, with a loss of less than $5 \%$ for $95 \%$ of the PMTs.

The long-term evolution of A/P is described by a model characterizing the exponential de- 

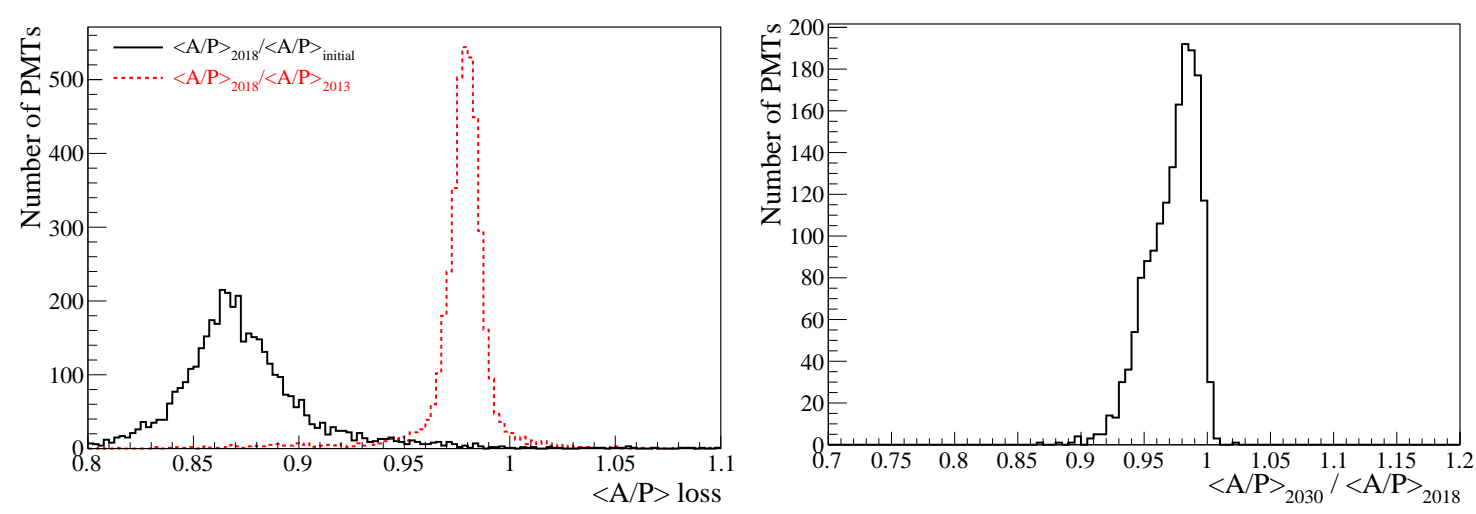

Figure 3: Left: Stacked distribution of $A / P$ loss, defined as $\langle A / P\rangle_{2018}$ divided by $\langle A / P\rangle_{\text {initial }}$ (solid, black) and by $\langle A / P\rangle_{2013}$ (dashed, red) of each PMT. Right: $\langle A / P\rangle$ expected in 2030 divided by $\langle A / P\rangle_{2018}$.
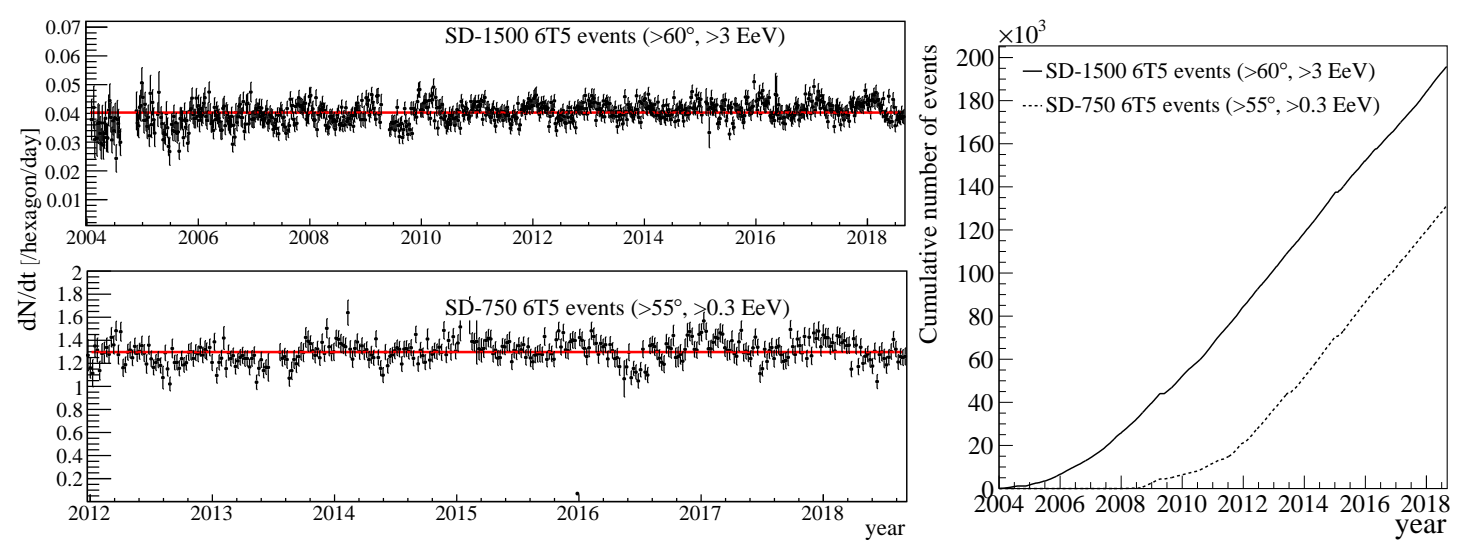

Figure 4: Left: Evolution of the daily 6T5 rate normalized to the number of hexagons. Top: Number of the SD-1500 array events with energy $>3 \times 10^{18} \mathrm{eV}$ and zenith angle $<60^{\circ}$. Bottom: Number of the SD750 array events with energy $>0.3 \times 10^{18} \mathrm{eV}$ and zenith angle $<55^{\circ}$. Red lines indicate the averages (see text). Note that the bin size is chosen to be a week, and error bars represent the corresponding statistical uncertainties. Right: Cumulative number of 6T5 events for the SD-1500 array (solid) and the SD-750 array (dashed).

cay combined with an annual modulation [6]. The A/P evolution is extrapolated to estimate the expected $\mathrm{A} / \mathrm{P}$ in the future. By selecting PMTs with having data for more than five months and without a discontinuity in the most recent two years of data, 1655 PMTs are fitted. As a result, $85 \%$ of these PMTs are expected to have an A/P larger than $95 \%$ of their current values in 2030 (Fig. 3). Based on this extrapolation, we can assume that the SD stations will not experience a significant change in their behavior within the operation time of the Pierre Auger Observatory.

The air-shower trigger is formed by requiring a time coincidence of at least three neighbouring stations [5]. For accurate reconstruction, a trigger condition, named as 6T5 trigger, further ensures that showers are contained in the array, by requiring that at the time of the event all six neighbouring stations of the station with the highest signal are active. The 6T5 events are the basis of high-level analyses like the measurements of the energy spectrum [7]. Thus a stable 6T5 rate is crucial. For 

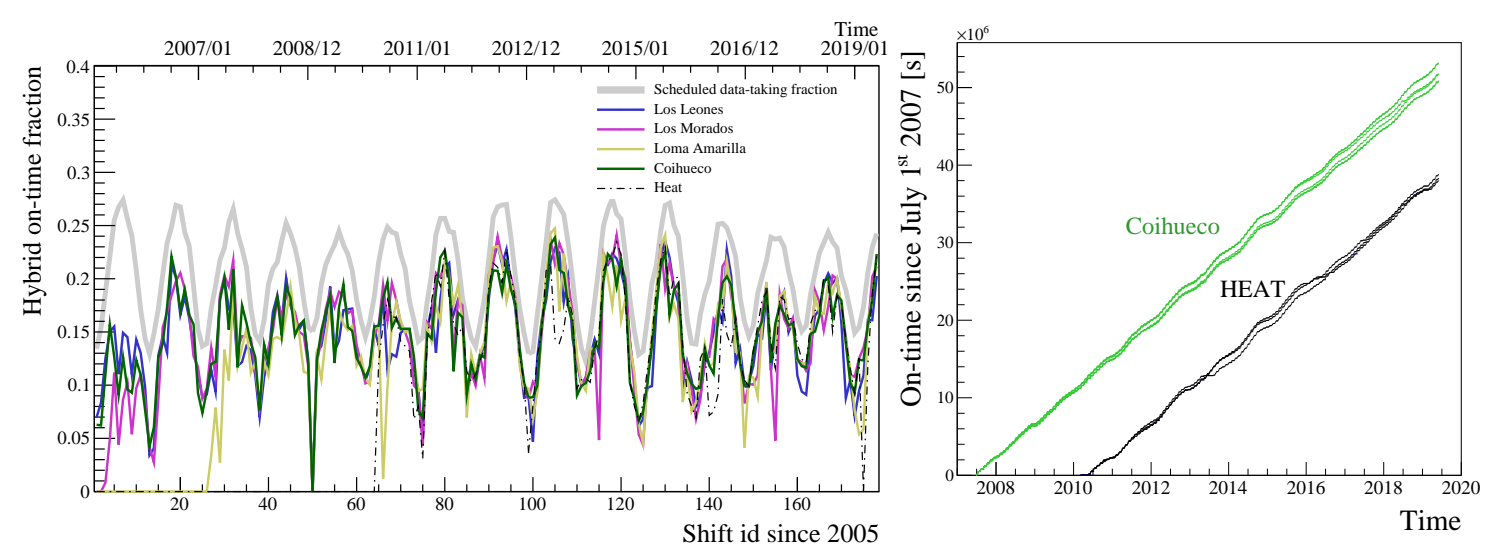

Figure 5: Left: The on-time fraction is shown for the four FD sites (Los Leones, Los Morados, Loma Amarilla and Coihueco) and the HEAT for hybrid events. The X-axis indicates shift ID, where each shift covers a moon cycle. Right: The accumulated on-time since July 1, 2007 for the six telescopes of Coihueco and the three telescopes of HEAT.

cosmic rays with zenith angle less than $60^{\circ}$ and energy larger than $3 \mathrm{EeV}$, the trigger efficiency for the SD-1500 array is array is close to 1, irrespective of the nature of the primary particle. For the SD-750 array, full efficiency is achieved above $0.3 \mathrm{EeV}$ and for zenith angle less than $55^{\circ}$. The time evolution of the daily rate of 6T5 events passing the full trigger efficiency conditions per hexagon is shown in Fig. 4 (left). The 6T5 rate has been constant over more than 14 years, being $0.040 \pm 0.004$ events/day/hexagon for the SD-1500 array and 1.3 \pm 0.1 events/day/hexagon for the SD-750 array. In 2013 new station triggers have been implemented which do not depend strongly on the shape of the signal. Being sensitive to small signals, they lower the energy threshold for full efficiency of the arrays, assuring a constant event rate above this threshold [8].

The cumulative number of 6T5 events is illustrated in Fig. 4 (right). The SD has been running with high efficiency of data accumulation throughout the 15 years of the data-taking history. The SD-1500 array data sets currently contain more than 4.5 million 6T5 events out of which more than 200,000 events are above $3 \mathrm{EeV}$.

\section{Long term performance of the Fluorescence Detector}

The fluorescence telescopes operate during dark, clear nights. Data taking requires the sun to be lower than $18^{\circ}$ below the horizon (evening and morning astronomical twilights) and the moon to be below the horizon for longer than three hours. In addition, the illuminated moon fraction has to be lower than 70\%. The sky background photon flux (Night Sky Brightness) and the artificial lights in the field of view of the FD telescopes should also be marginal. The data taking can also be affected by bad weather conditions such as rain, snow, strong winds and lightning. The weather conditions and the background noise (electronic and Night Sky Brightness) for the FD are continuously monitored during the data taking $[9,10]$.

When an air shower is observed by the FD in coincidence with at least one triggered SD station, a better reconstruction of the shower geometry is achieved with respect to the one using the FD alone [11]. These events are called hybrid events. Knowing the hybrid on-time is crucial 


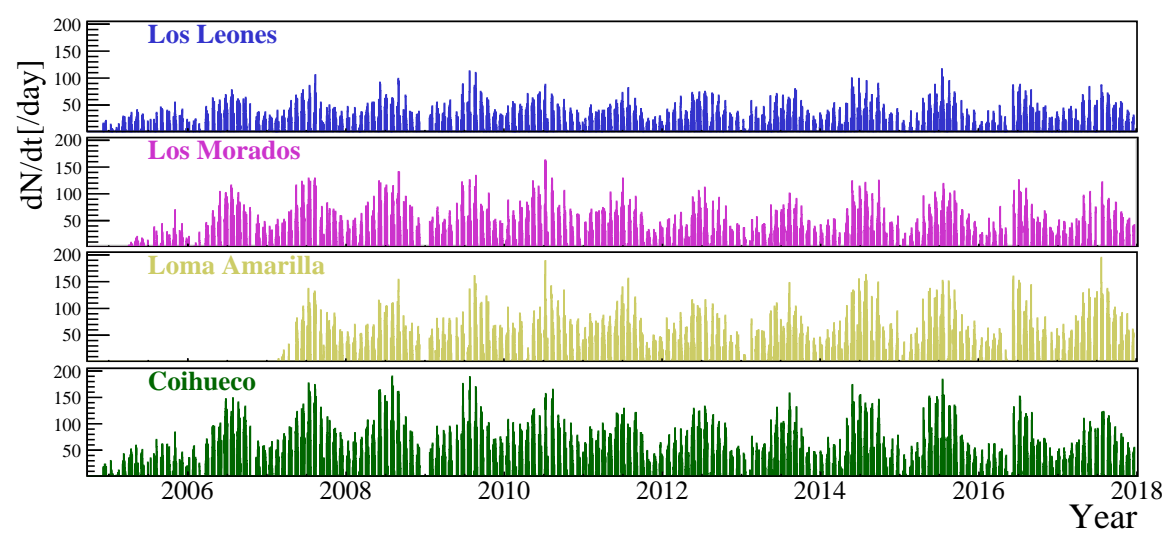

Figure 6: Number of events taken in each day are shown for the four FD sites, respectively.

for an accurate evaluation of the FD hybrid exposure [11]. The time evolution of the hybrid ontime fraction is shown in Fig. 5 (left) for four FD sites and the HEAT telescopes. The on-time of the hybrid detector is compared to the nominal Data Acquisition (DAQ) time fraction calculated according to the Sun and the Moon positions as previously described. In addition to the Night Sky Brightness and the weather conditions, technical problems such as DAQ failure can cause inefficiency of the FD operation. Excluding the initial phase when the telescopes were not fully operational, the on-time has been about $15 \%$ for all FD sites. Also, seasonal modulations are visible due to longer nights during winter. On the right side of Fig. 5, the accumulated on-time in seconds since July 1, 2007, for Coihueco and HEAT telescopes is shown. The stability of the hybrid detector is shown in Fig. 6 in terms of the daily number of hybrid events observed by individual FD sites with the same seasonal modulations being visible as in the on-time plot (Fig. 5).

Different methods are used to calibrate the FD. Absolute calibrations have been performed over the years to follow the long-term behavior, while a relative calibration is performed daily to follow the short- and medium-term changes due to different contributions, i.e. filters, mirrors, and PMTs. The absolute end-to-end calibration [12] used a drum-shaped tool, in which a pulsed UV-LED source illuminates the interior of a $2.5 \mathrm{~m}$ diameter cylindrical drum of $1.4 \mathrm{~m}$ depth, that can be mounted at the entrance aperture of each telescope. Three different relative calibration measurements [13], using light sources injected at three different positions on each telescope, are performed twice per night, before and after the FD data acquisition, to track daily variations as well as variations between two consecutive absolute calibrations. As an example, the mean of the calibration constants from all PMTs of telescope 3 at the Loma Amarilla site are shown in Fig. 7 (left) for the period from 2007 to 2019. A stable behaviour is reported after an initial drift [14].

Monitoring the atmospheric properties around the air-shower development is also critical for a reconstruction of the shower energies. Especially atmospheric transmission through aerosols needs rigorous monitoring due to their large and fast time variation and the significant effect on airshower reconstruction [1]. The Central Laser Facility (CLF) and the eXtreme Laser Facility (XLF) continuously take data to measure the aerosol optical depth vertical profiles in the FD field of view on an hourly basis [15]. During FD ãĚąėíĂ data acquisition, the two facilities vertically shoot a set of 50 collimated UV laser pulses every 15 minutes, which can be simultaneously detected by 

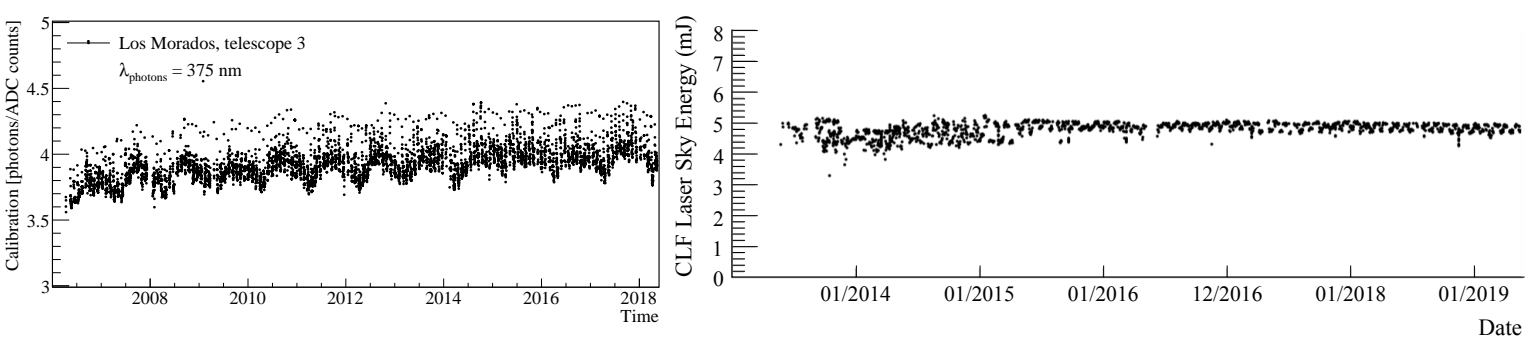

Figure 7: Left: Mean of the calibration constants from all PMTs of telescope 3 in the Loma Amarilla site, taken in each shift. Right: The CLF laser energy as a function of time.

different FD telescopes. The CLF has been operating since 2003, and a major upgrade was done in 2013 to add a beam calibration system and a backscatter Raman lidar receiver. For absolute calibrations of the CLF beam, the entire laser beam is sent to the sky and captured with an external radiometer before and after each night's operation. Fig. 7 (right) illustrates the stability of the energy of the CLF beam measured by the calibration system. For more information about the CLF and XLF facilities and the analyses refer to $[1,9,15]$.

\section{Conclusion}

We have presented the long term performance of the Pierre Auger Observatory over more than 15 years of data-taking history. The SD has been functioning stably with a duty cycle higher than $95 \%$. The study carried out on atmospheric muons shows that the SD have stabilized, the area over peak loss is expected to be less than 5\% for most of the PMTs in the next decade. The FD, as well as the instruments for calibration and monitoring of the detectors, have also been operating in a stable and efficient manner.

\section{References}

[1] The Pierre Auger Collaboration, Nucl. Instrum. Meth. A 798 (2015)

[2] The Pierre Auger Collaboration, arXiv:1604.03637 [astro-ph.IM]

[3] X. Bertou et al. for the Pierre Auger Collaboration, Nucl. Instrum. Meth. A 568 (2006)

[4] C. Bonifazi for the Pierre Auger Collaboration, ICRC (2013) 1079

[5] The Pierre Auger Collaboration, Nucl. Instrum. Meth. A 613 (2010)

[6] R. Sato for the Pierre Auger Collaboration, ICRC (2011) 204

[7] V. Verzi for the Pierre Auger Collaboration, PoS(ICRC2019) 450

[8] A. Coleman [Pierre Auger Collaboration], PoS(ICRC2019) 225

[9] The Pierre Auger Collaboration, Astropart. Phys. 33, 108 (2010)

[10] M. Kleifges, et al., IEEE Trans. Nucl. Sci. 50, 1204 (2003)

[11] P. Abreu, et al., Astropart. Phys. 34, 368 (2011)

[12] Brack J. et al., Astropart. Phys. 20, 653 (2004)

[13] R. Caruso for the Pierre Auger Collaboration, ICRC (2009) 473

[14] G. Salina for the Pierre Auger Collaboration, PoS(ICRC2015) 594

[15] V. Harvey for the Pierre Auger Collaboration, PoS(ICRC2019) 283 